\title{
BMJ Open Comparing the variants of takotsubo syndrome: an observational study of the ECG and structural changes from a New Zealand tertiary hospital
}

\author{
George M Watson, ${ }^{\oplus 1}$ Christina W Chan, ${ }^{1}$ Laura Belluscio, ${ }^{2}$ Kit Doudney, ${ }^{3}$ \\ Cameron J Lacey, ${ }^{4}$ Martin A Kennedy, ${ }^{5}$ Paul Bridgman ${ }^{1}$
}

To cite: Watson GM, Chan CW, Belluscio L, et al. Comparing the variants of takotsubo syndrome: an observational study of the ECG and structural changes from a New Zealand tertiary hospital. BMJ Open 2019;9:e025253. doi:10.1136/ bmjopen-2018-025253

- Prepublication history for this paper is available online. To view these files, please visit the journal online (http://dx.doi. org/10.1136/bmjopen-2018025253).

Received 5 July 2018 Revised 17 March 2019 Accepted 28 March 2019

\section{Check for updates}

C Author(s) (or their employer(s)) 2019. Re-use permitted under CC BY-NC. No commercial re-use. See rights and permissions. Published by BMJ.

${ }^{1}$ Cardiology, Christchurch Hospital, Christchurch, New Zealand

${ }^{2}$ Biostatistics, Christchurch Hospital, Christchurch, New Zealand

${ }^{3}$ Molecular Pathology, Canterbury District Health Board, Christchurch, New Zealand ${ }^{4}$ Psychological Medicine, Christchurch Hospital, Christchurch, New Zealand ${ }^{5}$ Department of Pathology, University of Otago,

Christchurch, Christchurch, New Zealand

Correspondence to Dr Paul Bridgman; paul.bridgman@cdhb.health.nz

\section{ABSTRACT}

Objectives In takotsubo syndrome, QTc prolongation is a measure of risk of potentially fatal arrhythmia. It is not known how this risk, or derangement of other markers, differs across the echo variants of takotsubo syndrome. Therefore, we sought to explore whether apical takotsubo syndrome differs from the variants of the syndrome in more ways than just regional wall motion pattern. As the region of affected myocardium is usually larger, we hypothesised that patients with the classic apical ballooning form of takotsubo syndrome would have more severe derangement of their markers.

Design Observational study of patients gathered from a prospective database (2010-2018) and by retrospective review (2006-2009).

Setting The sole tertiary hospital from a New Zealand region in which case clusters of takotsubo syndrome were precipitated by large earthquakes in 2010, 2011 and 2016 Participants A total of 222 patients who met a modified version of the Mayo criteria for takotsubo syndrome were included. All patients had digitally archived echocardiograms that were over-read by a second echocardiologist blinded to the clinical report.

Primary outcome measures Ejection fraction, peak troponin and QTc interval.

Results Patients with the apical form were older $(p=0.011)$, had a lower initial left ventricular ejection fraction ( $35 \%$ vs $44 \%, p<0.0001)$ and a higher peak high-sensitivity troponin I (hsTnl) $(p=0.01)$ than those with variant forms. There was no difference in the electrical abnormalities between the variants (QTC interval, heart rate, PR interval, QRS duration or T-wave axis). There was also no correlation between any of peak hsTnl, peak QTC and ejection fraction. QTc interval increased on day 2 and peaked on day 3 before falling steeply $(p<0.0001)$.

Conclusions The variants of takotsubo syndrome differ in more ways than just their echo pattern but do not differ in their electrical abnormalities. There is a dissociation between the structural and electrical abnormalities. QTC peaks on day 3 and then falls steeply.

\section{INTRODUCTION}

The reported incidence of takotsubo syndrome is increasing. ${ }^{1}$ It is agreed that even in the most obvious of cases we do not
Strengths and limitations of this study

- A key strength of this study is its size - this is one of the largest single-centre studies to date in takotsubo syndrome research.

- The data set is remarkably complete. The lack of missing data allows for robust comparisons and correlations, and increases confidence in our conclusions.

- It is a weakness of our study that we did not include late imaging results or other modalities, such as MRI.

- Not all patients were followed up until QTC normalised-future studies should continue serial recording of ECGs for a longer period to establish the later time course.

understand how the psychological stress triggers the heart illness. ${ }^{2}$ Cardiologists recognise that there are variants within the syndrome. The variants are defined by the specific pattern of wall motion abnormality in the left ventricle. We have noted how heterogeneous the clinical presentation of the condition is and that different subsets of patients can behave in different ways. Takotsubo syndrome has an early mortality rate comparable to that of an acute coronary syndrome. ${ }^{3}$ Characterising the differing subsets will be important in helping us to move towards an improved understanding of the condition and could lead to better-tailored management of patients and perhaps insights into aetiology. Therefore, we sought to explore whether the echocardiographic variants of the syndrome differ in more ways than just their regional wall motion pattern.

In everyday clinical practice, clinicians use left ventricular ejection fraction (LVEF) as a measure of risk and the degree of troponin elevation as an indicator of myocardial loss. Less widely recognised in takotsubo syndrome 
is that QTc interval is a measure of risk of potentially fatal arrhythmia. ${ }^{4}$ It is not known if this risk varies across the echo patterns of the condition. A report from the International Takotsubo (InterTAK) Registry suggests that outcome does not vary between the apical and variant patterns, whereas the other clinical, biochemical and electrical features that cardiologists deal in do. ${ }^{5}$ InterTAK found that patients with the typical takotsubo pattern had lower ejection fractions and higher brain natriuretic peptide levels than those with an atypical pattern. This is consistent with the observation that the region of affected myocardium is usually larger in typical patients. Outcome has previously been suggested to be adversely impacted by the presence of coexisting coronary artery disease. ${ }^{6}$

Our objective in this study was to explore whether apical takotsubo syndrome differs from the variants of the syndrome in more ways than just the regional wall motion pattern. We hypothesised that patients with the classic apical ballooning form of takotsubo syndrome would have more severe derangement of markers of myocardial involvement and more severe QT prolongation than patients with one of the variant forms.

\section{METHODS}

Christchurch Hospital is the only tertiary hospital in Canterbury, New Zealand. Unprecedented case clusters of takotsubo syndrome were precipitated by large earthquakes in Canterbury in 2010, 2011 and 2016, leading to the development of a prospectively maintained registry of cases since $2010 .{ }^{78}$ In addition, we retrospectively identified cases extending back to the installation of a digital echocardiography archive in 2006.

All patients in the database meet a modified form of the Mayo criteria for takotsubo syndrome, which allows inclusion of patients with one of the recognised characteristic patterns of wall motion abnormality. ${ }^{3}$ Patients are not excluded if they have coronary artery disease that does not explain their wall motion abnormality, or if they die before resolution can be documented on follow-up echo. All echocardiograms are held in a digital archive and were over-read by a second echocardiologist to confirm the wall motion pattern and the measurements. Discrepancies in the reporting were settled by joint review.

High-sensitivity troponin I (hsTnI) was used as a marker of myocardial damage. Prior to 2011, Christchurch Hospital used a standard TnI assay-this was converted to hsTnI using the conversion factor hsTnI $=880 \times \operatorname{TnI}+1096$, which was derived from a comparative study of the assays done at the Canterbury Health Laboratory.

The ECG machine performs automatic electronic measurement for heart rate (HR), PR interval, QRS width and QT interval. These were all checked by manual measurement. Where the manual measurement showed that the electronic measure was incorrect, the manual measure was used. Borderline discrepancies were resolved by third-party measurement. QTc interval was calculated using Bazett's formula. The ECG machine also calculates
T-wave axis. For this, manual measurement and calculation were not performed, and the electronic measure was always used. LVEF, hsTnI and ECG measurements were compared between the apical and variant (basal, focal and midwall) forms, and between days over a 4-day period from the time of admission.

Normality was assessed using Shapiro-Wilk test. Means of normally distributed variables were compared using the two-sample t-test, while the medians of variables that do not follow a normal distribution were compared by Wilcoxon rank-sum test. Non-parametric analysis of rank correlation was done using Kendall's tau.

\section{Patient and public involvement}

Patients and the public were not involved in the design of this study.

\section{RESULTS}

This study included 222 cases of takotsubo syndrome. Four of the patients were male. The echocardiographic pattern was apical in $178(80 \%)$ and variant in $44(20 \%)$. Among the variant patients, that pattern was midwall in 32 , inverted in 4 and focal in 8 . In 37 cases (17\%), the identified stressor was an earthquake. Another identified emotional stressor was present in 75 patients $(34 \%)$. There was a physical illness trigger in 38 patients (17\%), and in 7 cases (3\%), there was a concurrent emotional and physical trigger. There was no identified stressor in 65 cases (29\%). Presentation rhythm was atrial fibrillation in eight patients. At the time of admission, $9 \%$ of patients were on medications that may prolong the QT interval and have a definite or possible risk of ventricular arrhythmia. The rate of prescription of these medications was similar between patients with the apical type $(10 \%)$ and patients with a variant $(7 \%)$.

Overall, the patients with the apical form of the syndrome were significantly older (68 vs 64 years, $\mathrm{p}=0.011$ ). However, unlike the variants, there appeared to be a bimodal age distribution in the apical takotsubo patients. This is illustrated in figure 1 and suggests that there may be a distinct younger subset. We noted that all these younger patients had major psychological stressors, and in four cases, there was also a concomitant physical stressor.

Patients with the apical form of the syndrome had a lower initial LVEF (35\% vs 44\%, $\mathrm{p}<0.0001$ ), and a higher peak hsTnI $(\mathrm{p}=0.01)$ than those with variant forms, but there was no difference between the forms in peak QTc interval $(\mathrm{p}=0.93)$ (table 1). Apical and variant forms also did not differ significantly in the other ECG measures.

HR, QTc interval and T-wave axis all changed significantly with time (all $\mathrm{p}<0.0001$ ), but not by echo variant. QRS interval and PR interval did not differ by day or echo variant. There were no associations between the structural and electrical measures (table 2). While peak QTc against LVEF was borderline significant $(\mathrm{p}=0.04)$, the Kendal Tau correlation was only -0.097 . This is a very 


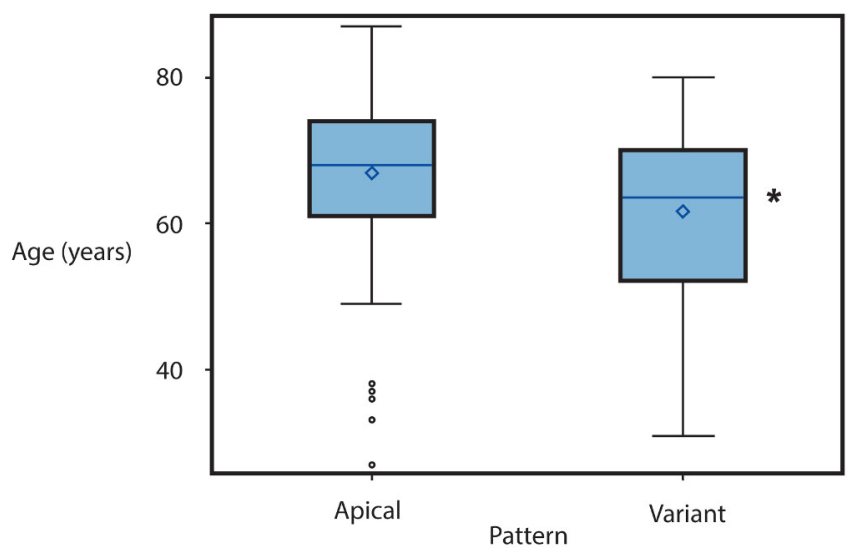

Figure 1 Comparison of age for patients with apical takotsubo versus variant pattern. The horizontal line in the middle of the box represents the median of each group, while the diamonds represent the mean. The box upper and lower bounds represent the upper and lower quartiles, respectively. The whiskers represent the minimum and maximum values excluding those that are $1.5 \times \mathrm{IQR}$ away from the median (circles). ${ }^{*} \mathrm{P}=0.011$.

weak correlation, and with marginal statistical significance is not meaningful, as demonstrated by the scatter plot (figure 2). We documented different time courses for the changes in QTc and troponin, and that QTc peaks on day 3 (figure 3 ).

\section{DISCUSSION}

We have found that the differences between the variants of takotsubo syndrome go beyond the echo features. Patients with the apical form are older and have a lower ejection fraction, corroborating findings reported by the InterTAK Registry. ${ }^{35}$ Patients with the apical form also tended to have a higher peak troponin. Of particular interest in our study is that QTc interval does not track with our structural indices (LVEF and peak troponin) and that the severity of QTc prolongation does not differ between the variants. We do not fully understand the pathophysiology of either the myocardial dysfunction or the QTc prolongation in takotsubo syndrome. However, we note that in pluripotent stem cell-derived cardiomyocytes, oestradiol
Table 2 Associations between LVEF, peak hsTnl and peak QTC

\begin{tabular}{lll}
\hline & $\begin{array}{l}\text { Correlation } \\
\text { coefficient }\end{array}$ & P value \\
\hline Peak hsTnl versus LVEF & -0.091 & 0.0609 \\
Peak hsTnl versus peak & -0.022 & 0.6498 \\
QTc & & \\
Peak QTc versus LVEF & -0.097 & 0.0457 \\
\hline
\end{tabular}

hsTnl, high-sensitivity troponin I; LVEF, left ventricular ejection fraction.

has been shown to protect against action potential prolongation, suggesting that reduction in oestrogen level may be a factor in increasing the risk of QT prolongation in these patients, who are predominantly postmenopausal. ${ }^{9}$ We also note that MRI of 20 patients with apical takotsubo has found that QTc prolongation may correlate with an apical to basal gradient seen on T2-weighted sequences for myocardial oedema. ${ }^{10}$ Our data, however, suggest that the repolarisation changes are not dependent on the degree of myocardial involvement. We speculate that the mechanisms of electrical and structural derangement must be separate from early in the pathophysiological process that follows the triggering event. This is an area that will require future research.

We have demonstrated a dissociation between the structural and electrical features in both severity and time course, including demonstrating that QTc peaks on day 3. A case series from Auckland found QTc rises until day 3 but did not look beyond that point and so did not capture a peak. ${ }^{11}$ A small series of 93 patients from two hospitals in Boston had previously reported a peak on day $3{ }^{4} \mathrm{We}$ have now confirmed that finding in a much larger cohort. The Boston group also found that QTc correlates with the risk of ventricular arrhythmia. There is, therefore, a strong case for in-hospital monitoring of patients while QTc is prolonged. It is important that patients are not discharged after a normal angiogram if their troponin is falling, without attention to their ECG and QTc interval. We have shown that typically QTc falls steeply between

Table 1 Comparison of LVEF, hsTnl and ECG measures between apical and variant takotsubo

\begin{tabular}{llll}
\hline & Apical takotsubo & Variant takotsubo & P value \\
\hline Initial LVEF (\%) & $36.67($ SEM 0.69) & $43.52($ SEM 1.60) & $<0.0001$ \\
Peak hsTnl (ng/L) & $2308(1244-3936)$ & $1638(289-2441)$ & 0.01 \\
Heart rate (bpm) & $79(70-94)$ & $76(66-95)$ & 0.18 \\
PR interval (ms) & $166(150-178)$ & $165(143-180)$ & 0.49 \\
QRS duration (ms) & $88(82-100)$ & $88(80-96)$ & 0.90 \\
QTc interval (ms) & $465(434-490)$ & $459(433-484)$ & 0.93 \\
T-wave axis & $75(54-106.5)$ & $76(52-98)$ & 0.95 \\
\hline
\end{tabular}

All ECG variables are as at presentation. LVEF is mean and SE, and other data are median and IQR.

hsTnl, high-sensitivity troponin I; LVEF, left ventricular ejection fraction; SEM, standard error of the mean. 


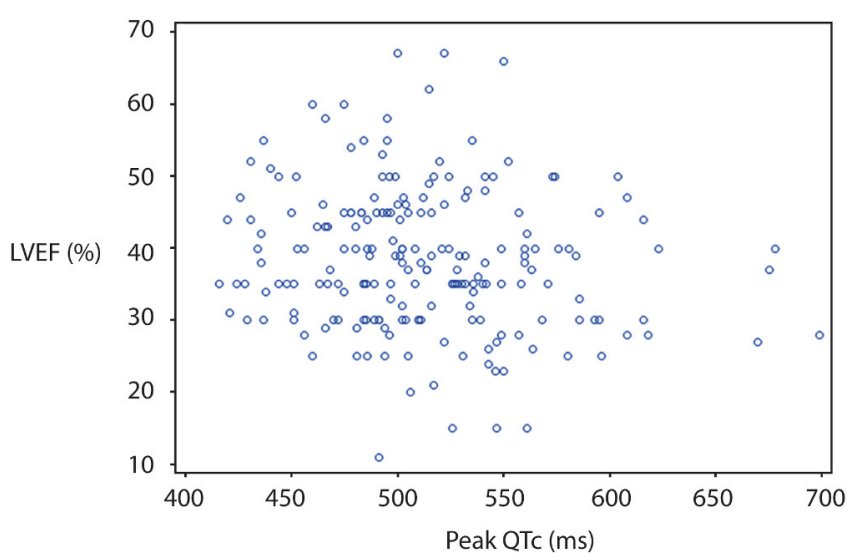

Figure 2 Scatter plot showing the correlation between peak QTc interval and LVEF. LVEF, left ventricular ejection fraction.

days 3 and 4 . The implication of this for clinicians is that daily ECGs should be performed until the QTc has normalised and that patients should not be discharged early.

Our data highlight the dissociation of the structural and electrical features and a complex relationship between the structural indices. While the apical form typically presents with a higher troponin level and a lower ejection fraction, there is no relationship between these two. This suggests that they are reflecting different aspects of the condition. Typically, in apical takotsubo, a very large area of myocardium is initially akinetic and while troponin does rise, the rise is relatively small, reflecting that only a very low fraction of affected cells are dying. On standard 2D echo, the vast majority of patients have a full-function recovery. However, a study of 52 patients from Scotland has found that on advanced echo and MRI studies it is possible to detect residual effects and permanent damage. ${ }^{12}$ Our results are consistent with the concept that the degree of acute troponin rise reflects acute cell death and that the number of cells affected in this way is uncorrelated with the total area of myocardium affected.

Our finding that variant patients are on average younger is not new and has previously been reported by the InterTAK Registry. ${ }^{35}$ However, these reports did not explore the distribution of age with each variant. In our cohort, there appeared to be a bimodal distribution among the apical variant patients with the younger patients having the same syndrome (comparable LVEF, hsTnI and QTc prolongation) as the older patients, but usually with dual or very severe precipitants. This suggests that there is a higher threshold for triggering the apical syndrome in this group of younger patients.

Features of our study population suggest that the study results should have good external validity. In our cohort, $80 \%$ of cases were apical takotsubo and $20 \%$ were variant, similar to the InterTAK Registry cohort. In both our study population and in the InterTAK Registry, the patients with a variant pattern tended to be younger. ${ }^{35} \mathrm{~A}$ minor difference between our case population and other case series is in the ratio of males to females. Other multicentre registries have reported that males account for $5 \%-15 \%$ of cases. ${ }^{1314}$ In the current study, $2 \%$ of cases are male. The major clinical implication of our study is that QT prolongation peaks late and therefore, daily ECGs are required, and inpatient care should not be shortened. In the absence of contrary evidence, we believe that it is prudent that males are treated in this manner also.

It is a weakness of our study that we do not have follow-up MRI data. Future work should look to correlate long-term

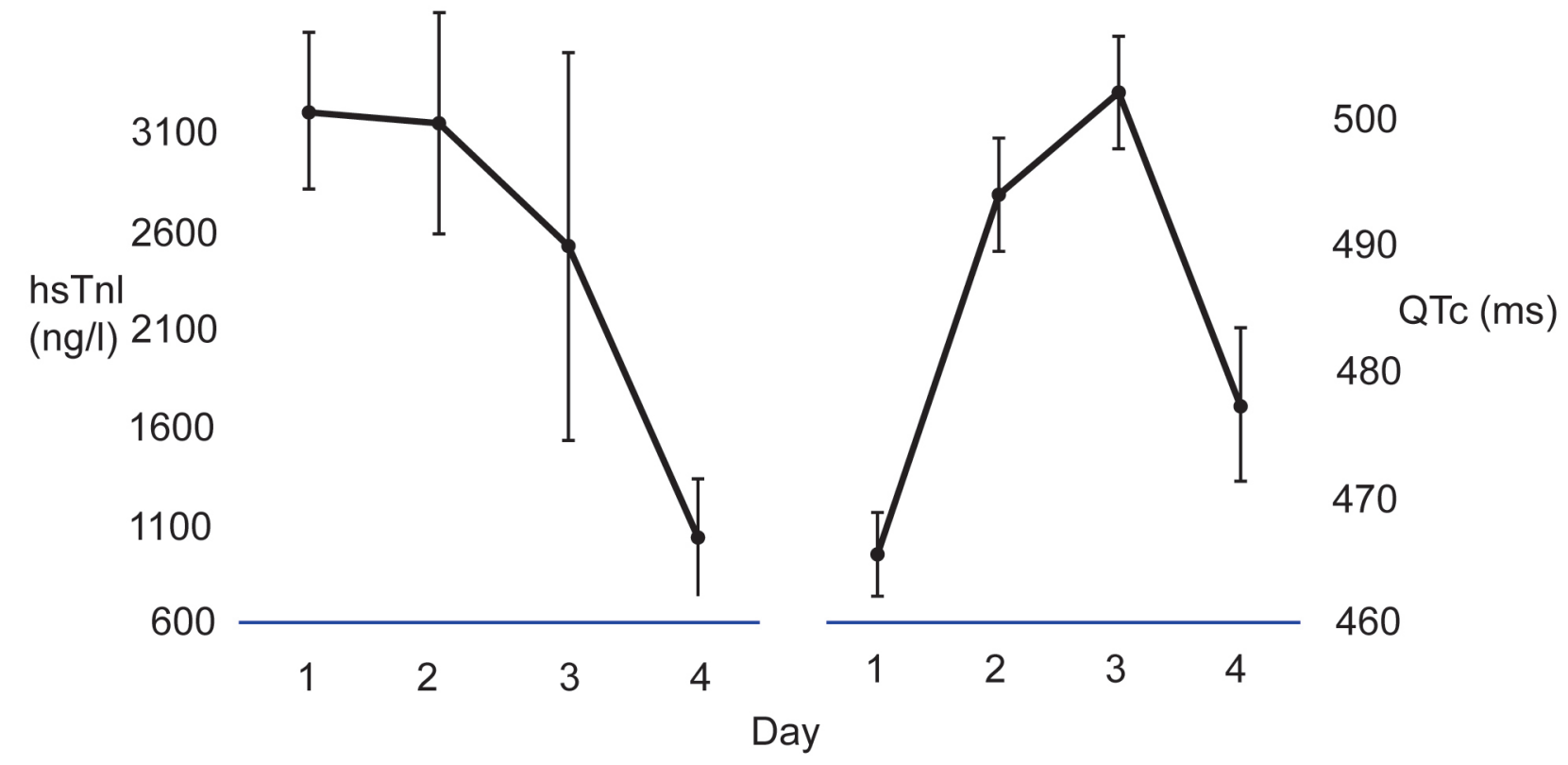

Figure 3 Change in hsTnl and QTc interval over time after admission. hsTnl, high-sensitivity troponin I. 
changes with the acute indices of syndrome severity. Our data show a steep fall in QTc from day 3 to 4, but further studies should also continue serial recording of ECGs for a longer period to establish the later time course.

\section{CONCLUSIONS}

The variants of takotsubo syndrome differ in more ways than just their echo pattern but do not differ in their electrical abnormalities. There is a dissociation between the structural and electrical abnormalities. QTc peaks on day 3 and then falls steeply.

Contributors The authors contributed to the study as follows: design and conception (PB, MAK and CJL), acquisition of data (GMW and CWC), and data interpretation and manuscript preparation (GMW, CWC, LB, KD, CJL, MAK and PB).

Funding GMW was funded as a University of Otago Summer Student by the National Heart Foundation of New Zealand.

Competing interests No, there are no competing interests for any author. Patient consent for publication Not required.

Ethics approval The study was approved by the relevant statutory authority in New Zealand, the Health and Disability Ethics Committee. The approval number is URA/11/07/033/AM03.

Provenance and peer review Not commissioned; externally peer reviewed.

Data sharing statement For this study, we used data and ECGs collected during routine clinical practice. All relevant summary data are provided in the paper. The Canterbury District Health Board does not grant permission for the raw data to be made publically available due to ethical and legal restrictions. However, all interested readers may request data without restriction from PB (paul.bridgman@ cdhb.health.nz).

Open access This is an open access article distributed in accordance with the Creative Commons Attribution Non Commercial (CC BY-NC 4.0) license, which permits others to distribute, remix, adapt, build upon this work non-commercially, and license their derivative works on different terms, provided the original work is properly cited, appropriate credit is given, any changes made indicated, and the use is non-commercial. See: http://creativecommons.org/licenses/by-nc/4.0/.

\section{REFERENCES}

1. Minhas AS, Hughey AB, Kolias TJ. Nationwide trends in reported incidence of takotsubo cardiomyopathy from 2006 to 2012. Am J Cardiol 2015;116:1128-31.

2. Otten AM, Ottervanger JP, Symersky T, et al. Diagnosis of takotsubo cardiomyopathy is increasing over time in patients presenting as STelevation myocardial infarction. Neth Heart J 2016;24:520-9.

3. Templin C, Ghadri JR, Diekmann J, et al. Clinical features and outcomes of takotsubo (stress) cardiomyopathy. N Engl J Med 2015;373:929-38.

4. Madias C, Fitzgibbons TP, Alsheikh-Ali AA, et al. Acquired long QT syndrome from stress cardiomyopathy is associated with ventricular arrhythmias and torsades de pointes. Heart Rhythm 2011;8:555-61.

5. Ghadri JR, Cammann VL, Napp LC, et al. Differences in the clinical profile and outcomes of typical and atypical takotsubo syndrome. JAMA Cardiol 2016;1:335-40.

6. Bill V, El-Battrawy I, Schramm K, et al. Coincidental coronary artery disease impairs outcome in patients with takotsubo cardiomyopathy. QJM 2017;110:483-8.

7. Lacey C, Mulder R, Bridgman P, et al. Broken heart syndrome - is it a psychosomatic disorder? J Psychosom Res 2014;77:158-60.

8. Chan C, Troughton R, Elliott J, et al. One-year follow-up of the 2011 Christchurch Earthquake stress cardiomyopathy cases. N Z Med J 2014;127:15-22.

9. El-Battrawy I, Zhao Z, Lan H, et al. Estradiol protection against toxic effects of catecholamine on electrical properties in humaninduced pluripotent stem cell derived cardiomyocytes. Int J Cardiol 2018;254:195-202.

10. Perazzolo Marra M, Zorzi A, Corbetti F, et al. Apicobasal gradient of left ventricular myocardial edema underlies transient T-wave inversion and QT interval prolongation (Wellens' ECG pattern) in Tako-Tsubo cardiomyopathy. Heart Rhythm 2013;10:70-7.

11. Looi JL, Wong CW, Lee M, et al. Usefulness of ECG to differentiate takotsubo cardiomyopathy from acute coronary syndrome. Int $J$ Cardiol 2015;199:132-40.

12. Schwarz K, Ahearn T, Srinivasan J, et al. Alterations in cardiac deformation, timing of contraction and relaxation, and early myocardial fibrosis accompany the apparent recovery of acute stress-induced (takotsubo) cardiomyopathy: an end to the concept of transience. J Am Soc Echocardiogr 2017;30:745-55.

13. Tornvall P, Collste O, Ehrenborg E, et al. A case-control study of risk markers and mortality in takotsubo stress cardiomyopathy. J Am Coll Cardiol 2016;67:1931-6.

14. Shah M, Ram P, Lo KBU, Lo K, et al. Etiologies, predictors, and economic impact of readmission within 1 month among patients with takotsubo cardiomyopathy. Clin Cardiol 2018;41:916-23. 